Proceedings of SALT 23: 514-534, 2013

\title{
Measure semantics and qualitative semantics for epistemic modals*
}

\author{
Wesley H. Holliday \\ University of California, Berkeley
}

Thomas F. Icard, III

Stanford University

\begin{abstract}
In this paper, we explore semantics for comparative epistemic modals that avoid the entailment problems shown by Yalcin $(2006,2009,2010)$ to result from Kratzer's (1991) semantics. In contrast to the alternative semantics presented by Yalcin and Lassiter $(2010,2011)$ based on finitely additive measures, we introduce semantics based on qualitatively additive measures, as well as semantics based on purely qualitative orderings, including orderings on propositions derived from orderings on worlds in the tradition of Kratzer (1991, 2012). All of these semantics avoid the entailment problems that result from Kratzer's semantics. Our discussion focuses on methodological issues concerning the choice between different semantics.
\end{abstract}

Keywords: epistemic modality, measure semantics, finite additivity, qualitative additivity, relative likelihood, qualitative probability, ordering semantics, completeness theorems

\section{Introduction}

What is the relation between ordinary talk using epistemic modal expressions such as 'probably' and 'at least as likely as' and the mathematical theory of probability? Is Kolmogorovian probability implicated in the semantics of these expressions? Until recently, the answer was thought to be negative. In an early discussion of the epistemic modal 'probably', Hamblin (1959: 234) wrote: "Metrical probability theory is well-established, scientifically important and, in essentials, beyond logical reproof. But when, for example, we say 'It's probably going to rain', or 'I shall probably be in the library this afternoon', are we, even vaguely, using the metrical probability concept?" Hamblin thought not. Similarly, Kratzer (2012: 25) writes: "Our semantic knowledge alone does not give us the precise quantitative notions of probability and desirability that mathematicians and scientists work with."1

* While writing this paper, we benefited greatly from conversations with Dan Lassiter and Seth Yalcin. 1 Some probability theorists have expressed related sentiments. For example, Koopman (1940: 269270) wrote: "Now we hold that such a number is in no wise a self-evident concomitant with or expression of the primordial intuition of probability, but rather a mathematical construct derived from the latter under very special conditions and as a result of a fairly complicated process implicitly based 
Semantics for epistemic modals

However, for the semantics of comparative epistemic modals such as 'at least as likely as', accounts that eschew probability have so far proven problematic. Yalcin (2006, 2009, 2010) has shown that Kratzer's (1991) semantics, based on qualitative orderings on propositions derived from qualitative orderings on worlds, not only fails to validate some desirable inference patterns, but also validates some undesirable patterns, as does Hamblin's semantics based on possibility measures. By contrast, Yalcin shows that a semantics based on probability measures avoids these problems. Also motivated by entailment problems for the semantics in Kratzer 1991, 2012, Lassiter $(2010,2011,2013)$ proposes a similar probability-based semantics. With this background, the question naturally arises of whether probability is necessary for an adequate semantics of epistemic comparatives in natural language.

In this paper, we explore semantics that avoid the entailment problems that result from Kratzer's semantics, but that are not based on probability measures. After some logical preliminaries in $\S 2$ and $\S 3$, we introduce three kinds of models in $\S 4$, for measure semantics, event-ordering semantics, and world-ordering semantics. In §5, we review Kratzer's world-ordering semantics, and in $§ 6$, we review Yalcin and Lassiter's measure semantics based on finitely additive probability measures. We then turn to new semantics: in $\S 7$, we introduce a measure semantics based on qualitatively additive measures; in $\S 8$, we briefly discuss event-ordering semantics; and in $\S 9$, we introduce a new method of lifting orderings on worlds to orderings on propositions for world-ordering semantics. All of the new semantics avoid the entailment problems that result from Kratzer's semantics. In $§ 10$, we discuss methodological issues concerning the choice between different semantics.

\section{Formal setup}

To facilitate comparison of different semantics, we focus on a single formal language. Given a set At of atomic sentences, our language $\mathscr{L}$ is generated by the grammar

$$
\varphi::=p|\neg \varphi|(\varphi \wedge \varphi)|\nabla \varphi|(\varphi \geqslant \varphi)
$$

where $p \in$ At. The intended reading of $\varphi \geqslant \psi$ is ' $\varphi$ is at least as likely as $\psi$ '. We take $\vee, \rightarrow, \leftrightarrow, \perp$, and $\top$ to be abbreviations as usual, as well as the following:

$$
\begin{array}{ll}
\varphi>\psi:=(\varphi \geqslant \psi) \wedge \neg(\psi \geqslant \varphi) & \text { ' } \varphi \text { is more likely than } \psi \text { '; } \\
\triangle \varphi:=\varphi>\neg \varphi & \text { ' } \varphi \text { is more likely than not' or 'probably } \varphi \text { '. }
\end{array}
$$

The technical reading of $\nabla \varphi$ will be ' $\varphi$ is true at some epistemically possible world'; and defining $\square:=\neg \diamond \neg$, the technical reading of $\square \varphi$ will be ' $\varphi$ is true at all

on many of the very intuitive assumptions which we are endeavoring to axiomatize. There is, in short, what appears to us to be a serious rational lacuna between the primal intuition of probability, and that branch of the theory of measure which passes conventionally under the name probability." 
epistemically possible worlds'. For the fragment of $\mathscr{L}$ without $\geqslant$, we assume that models are standard relational structures $\mathscr{M}=\langle W, R, V\rangle$ where $W$ is a non-empty set of "worlds," $R$ is a binary "accessibility" relation on $W, V:$ At $\rightarrow \wp(W)$ is a valuation, and the assignment of propositions to expressions is as follows:

$$
\begin{aligned}
& \llbracket p \rrbracket^{\mathscr{M}}=V(p) ; \quad \quad \llbracket(\varphi \wedge \psi) \rrbracket^{\mathscr{M}}=\llbracket \varphi \rrbracket^{\mathscr{M}} \cap \llbracket \psi \rrbracket^{\mathscr{M}} ; \\
& \llbracket \neg \varphi \rrbracket^{\mathscr{M}}=W-\llbracket \varphi \rrbracket^{\mathscr{M}} ; \quad \llbracket \nabla \varphi \rrbracket^{\mathscr{M}} \quad=\left\{w \in W \mid R(w) \cap \llbracket \varphi \rrbracket^{\mathscr{M}} \neq \emptyset\right\},
\end{aligned}
$$

where $R(w)=\{v \in W \mid w R v\}$ is the set of worlds accessible from $w$. Hence,

$$
\llbracket \square \varphi \rrbracket^{\mathscr{M}}=\left\{w \in W \mid R(w) \subseteq \llbracket \varphi \rrbracket^{\mathscr{M}}\right\} .
$$

We say that $\varphi$ is valid over a class of models $\mathscr{C}$ iff for all models $\mathscr{M}=\langle W, R, V\rangle$ in $\mathscr{C}, \llbracket \varphi \rrbracket^{\mathscr{M}}=W$; and $\varphi$ is invalid iff it is not valid. We will restrict attention to the class of models in which $R$ is a serial relation, i.e., for all $w \in W, R(w) \neq \emptyset$. All of our results also easily extend to smaller model classes obtained by further constraints on $R$, e.g., the uniformity constraint that for all $w, v \in W, R(w)=R(v)$.

As for the intuitive reading of $\diamond \varphi$, according to Yalcin's (2010: 927) semantics, $\nabla \varphi$ would be read as 'it might be that $\varphi$ '. ${ }^{2}$ But according to Kratzer's (1991) semantics, the truth of 'it might be that $\varphi$ ' requires more than $\varphi$ being true at some epistemically possible world; as explained in $\S 5, \diamond \varphi$ in the semantics above is equivalent to $\varphi>\perp$ in Kratzer's semantics, i.e., $\varphi$ is more likely than a contradiction. As a result of these differences, we will stick with the technical reading of $\nabla \varphi$.

Our main question in this paper is: what should the semantics be for $\geqslant$ ?

The literature on comparative epistemic modals provides some desiderata for an adequate semantics to satisfy. Yalcin (2010) presents a list of intuitively valid patterns, V1-V12, as well as intuitively invalid patterns, I1-I3, which we list in a condensed form in Figure 1 below, along with the pattern V13, suggested to us by Daniel Lassiter (personal communication, Lassiter 2013). We can assess different semantic proposals according to whether they validate V1-V13 and other intuitively valid patterns, and invalidate I1-I3 and other intuitively invalid patterns. In addition to this case-by-case analysis of validities and invalidities, we think it is useful to identify complete logics for the different semantics, for reasons explained in $\S 3$.

\section{Completeness theorems: what and why?}

When the semanticist offers a model-theoretic treatment of some fragment of language, this gives rise to predictions about logical relationships among expressions of

2 The "domain semantics" for $\mathscr{L}$ in Yalcin 2007, 2010 is equivalent to the relational semantics above assuming the uniformity constraint. See Holliday \& Icard 2013b for further discussion of this issue and of various definitions of consequence considered by Yalcin and others for languages with modals. 
Semantics for epistemic modals

$\begin{array}{llll}\text { V1 } & \triangle \varphi \rightarrow \neg \triangle \neg \varphi & \text { V11 } & (\psi \geqslant \varphi) \rightarrow(\triangle \varphi \rightarrow \triangle \psi) \\ \text { V2 } & \triangle(\varphi \wedge \psi) \rightarrow(\triangle \varphi \wedge \Delta \psi) & \text { V12 } & (\psi \geqslant \varphi) \rightarrow((\varphi \geqslant \neg \varphi) \rightarrow(\psi \geqslant \neg \psi)) \\ \text { V3 } & \triangle \varphi \rightarrow \triangle(\varphi \vee \psi) & \text { V13 } & ((\varphi \wedge \neg \psi)>\perp) \rightarrow((\varphi \vee \psi)>\psi) \\ \text { V4 } & \varphi \geqslant \perp & & \\ \text { V5 } & \top \geqslant \varphi & \text { I1 } & ((\varphi \geqslant \psi) \wedge(\varphi \geqslant \chi)) \rightarrow(\varphi \geqslant(\psi \vee \chi)) \\ \text { V6 } & \square \varphi \rightarrow \triangle \varphi & \text { I2 } & (\varphi \geqslant \neg \varphi) \rightarrow(\varphi \geqslant \psi) \\ \text { V7 } & \triangle \varphi \rightarrow \diamond \varphi & \text { I3 } & \triangle \varphi \rightarrow(\varphi \geqslant \psi)\end{array}$

Figure 1 List of intuitively valid (V1-V13) and invalid (I1-I3) patterns.

the language. An analysis is presumed adequate to the extent that these predictions accord with the intuitions of the common competent speaker of the language. In their popular textbook, Chierchia \& McConnell-Ginet (2001) explain the methodology:

We are capable of assessing certain semantic properties of expressions and how two expressions are semantically related. These properties and relationships and the capacity that underlies our recognition of them constitute the empirical base of semantics. (52)

These "semantic properties" include especially such logical concepts as entailment, validity, and contradiction. If a typical speaker finds some entailment pattern invalid, but the semantic analysis predicts it to be valid, that serves as evidence against the analysis. Conversely, if competent speakers find a pattern clearly valid, the semantic analysis will ideally predict that. While there are a number of unresolved issues concerning this methodology-e.g., Who counts as a competent speaker of a language?-we would like to raise a constructive methodological point.

Given a language and a class of models, in order to see what the predictions about entailment and validity in fact are, it is illuminating to prove completeness theorems. Such results isolate a few key principles from which all other validities and entailments can be derived, thus allowing a clearer view of what the relevant predictions are. Specifically, if we have a formal system $\mathbf{L}$, i.e., a set of axioms in our language together with rules of inference, then a soundness theorem with respect to some class $\mathscr{C}$ of models states that everything derivable from $\mathbf{L}$ is valid over $\mathscr{C}$. A completeness theorem states that if some expression is valid over $\mathscr{C}$, then it is derivable from $\mathbf{L}$. In what follows, we present a number of completeness results-some from the logic literature, some of our own results- that we believe shed light on recent work on epistemic modality. As a warm-up, below we state two standard completeness theorems for the basic semantics for $\diamond$ in $\S 2$. 


$$
\begin{array}{llll}
\text { Taut } & \text { all instances of tautologies } & \mathrm{MP} & \frac{\varphi \rightarrow \psi}{\psi} \\
\mathrm{RE} \diamond & \frac{\varphi \leftrightarrow \psi}{\diamond \varphi \leftrightarrow \diamond \psi} & \mathrm{N} \diamond & \neg \diamond \perp \\
\mathrm{M} \diamond & (\diamond \varphi \vee \diamond \psi) \rightarrow \diamond(\varphi \vee \psi) & \mathrm{C} \diamond & \diamond(\varphi \vee \psi) \rightarrow(\nabla \varphi \vee \diamond \psi)
\end{array}
$$

Figure 2 Logic $\mathbf{K}$ above. Logic $\mathbf{K D}$ is $\mathbf{K}$ plus the $\mathrm{D}$ axiom $\square \varphi \rightarrow \diamond \varphi$.

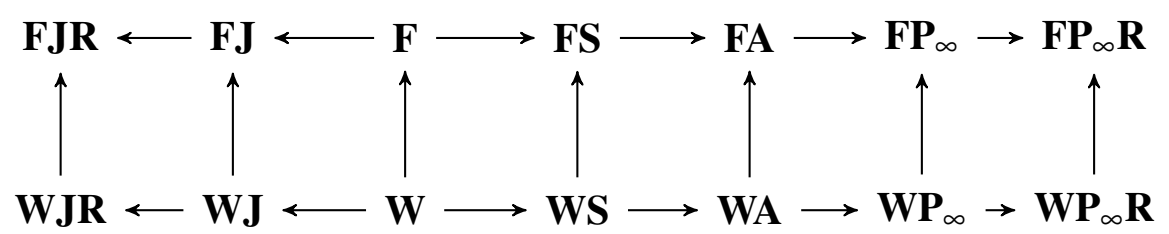

Figure 3 Logical landscape.

Theorem 1 The logic $\mathbf{K}$ in Figure 2 is sound and complete with respect to the class of relational models as in $\S 2$; and the logic $\mathbf{K D}$ in Figure 2 is sound and complete with respect to the class of serial relational models. ${ }^{3}$

Insofar as $\geqslant$ in our formal language corresponds to the ordinary English locution 'at least as likely as', the completeness results to be stated will provide succinct summaries of the validities predicted by alternative semantic accounts.

In a longer paper (Holliday \& Icard 2013b), we explore the logical landscape shown in Figure 3, where an arrow points from a $\operatorname{logic} \mathbf{L}$ to a $\operatorname{logic} \mathbf{L}^{\prime}$ iff every expression derivable from $\mathbf{L}$ is also derivable from $\mathbf{L}^{\prime}$; thus, the weakest logic is $\mathbf{W}$, and the two strongest logics, which are incomparable with each other, are FJR and $\mathbf{F P}_{\infty} \mathbf{R}$. In this paper we cover the logics WJR (§5), $\mathbf{F P}_{\infty} / \mathbf{F P} \infty \mathbf{R}(\S 6), \mathbf{F A}(\S 7)$, WA $(\S 8)$, and $\mathbf{W} \mathbf{P}_{\infty} \mathbf{R}(\S 9)$. Before discussing these logics, however, we must introduce our three main classes of models for $\geqslant$, subclasses of which give rise to these logics.

\section{Three kinds of models}

In this paper we will consider three kinds of models for epistemic comparatives. To define these models with convenient notation, given $P \subseteq W$, let $P_{w}=P \cap R(w)$. Thus, in the truth definitions below we can write $\llbracket \varphi \rrbracket_{w}^{\mathscr{M}}$ instead of $\llbracket \varphi \rrbracket^{\mathscr{M}} \cap R(w)$.

$3 \mathbf{K}$ is usually formulated with just one axiom and one rule (usually for $\square$ ), but the more fine-grained formulation in Figure 2 is equivalent (see Chellas 1980: Thms. 4.5, 5.12-14). Note that a rule like $\mathrm{RE} \diamond$ says that if the expression above the line has been derived as a theorem of the logic (and hence is valid, assuming the logic is sound), then the expression below the line can be derived as a theorem. 
Semantics for epistemic modals

In measure semantics, models are tuples $\mathscr{M}=\langle W, R, V, \mathscr{F}, \mu\rangle$ where:

- $\mathscr{F}$ is a function assigning to each $w \in W$ a $\sigma$-algebra $\mathscr{F}_{w}$ on $R(w)$, i.e., $\mathscr{F}_{w}$ is a set of subsets of $R(w)$ closed under complementation (relative to $R(w)$ ) and countably infinite unions (e.g., the power set algebra $\wp(R(w)))$;

- $\mu$ is a function assigning to each $w \in W$ a function $\mu_{w}: \mathscr{F}_{w} \rightarrow[0,1]$ such that $\mu_{w}(\emptyset)=0$ and $\mu_{w}(R(w))=1$;

- $\llbracket \varphi \geqslant \psi \rrbracket^{\mathscr{M}}=\left\{w \in W \mid \mu_{w}\left(\llbracket \varphi \rrbracket_{w}^{\mathscr{M}}\right) \geq \mu_{w}\left(\llbracket \psi \rrbracket_{w}^{\mathscr{M}}\right)\right\}$.

Special classes of measure models are obtained by adding further constraints on $\mu_{w}$.

In event-ordering semantics, models are tuples $\mathscr{M}=\langle W, R, V, \succsim\rangle$ where:

- $\succsim$ is a function assigning to each $w \in W$ a binary relation $\succsim_{w}$ on $\wp(R(w))$ such that for all $P \subseteq R(w), R(w) \succsim_{w} P \succsim_{w} \emptyset$;

- $\llbracket \varphi \geqslant \psi \rrbracket^{\mathscr{M}}=\left\{w \in W \mid \llbracket \varphi \rrbracket_{w}^{\mathscr{M}} \succsim_{w} \llbracket \psi \rrbracket_{w}^{\mathscr{M}}\right\}$.

Special classes of event-ordering models are obtained by adding constraints on $\succsim_{w}$.

In world-ordering semantics, there is a "lifting" operation $\uparrow$ that assigns to each relational model $\langle W, R, V\rangle$, world $w \in W$, and binary relation $\succeq_{w}$ on $R(w)$, a binary relation $\succeq_{w}^{\uparrow}$ on $\wp(R(w))$, and models are tuples $\mathscr{M}=\langle W, R, V, \succeq\rangle$ where:

- $\succeq$ is a function assigning to each $w \in W$ a preorder $\succeq_{w}$ on $R(w)$, i.e., a reflexive and transitive binary relation;

- $\llbracket \varphi \geqslant \psi \rrbracket^{\mathscr{M}}=\left\{w \in W \mid \llbracket \varphi \rrbracket_{w}^{\mathscr{M}} \succeq_{w}^{\uparrow} \llbracket \psi \rrbracket_{w}^{\mathscr{M}}\right\}$.

Special classes of world-ordering models are obtained by the choice of the operation $\uparrow$ and by adding constraints on $\succeq_{w}$.

\section{Kratzer's world-ordering semantics}

The first concrete semantics we consider is Kratzer's (1991) world-ordering semantics. In Kratzer's models, $\uparrow$ is what we call the $l$-lifting, due to Lewis (1973):

$$
A \succeq_{w}^{l} B \text { iff } \forall b \in B_{w} \exists a \in A_{w}: a \succeq_{w} b,
$$

recalling that for $P \subseteq W, P_{w}=P \cap R(w) .{ }^{4}$ As stated in $\S 4$, on such a world-ordering semantics, $\varphi \geqslant \psi$ is true at $w$ iff $\llbracket \varphi \rrbracket_{w}^{\mathscr{M}} \succeq_{w}^{l} \llbracket \psi \rrbracket_{w}^{\mathscr{M}}$. Also observe that on this

4 In Kratzer's (1991) semantics, $R$ and $\succeq$ are themselves derived from a "modal base" and an "ordering source." In Kratzer's notation, $R(w)$ is $\bigcap \mathrm{f}(w)$ and $\succeq_{w}$ is $\succeq_{\mathrm{g}(w)}$. Any $R$ and $\succeq$ can be obtained from some modal base and ordering source (Lewis 1981), so the valid principles according to Kratzer's semantics will be exactly the valid principles over the world-ordering models considered here. 


$$
\begin{aligned}
\text { BT } & \neg(\perp \geqslant \top) \\
\text { Tran } & (\varphi \geqslant \psi) \rightarrow((\psi \geqslant \chi) \rightarrow(\varphi \geqslant \chi)) \quad \text { Mon } \quad \square(\varphi \rightarrow \psi) \rightarrow(\psi \geqslant \varphi) \\
\mathrm{J} & ((\varphi \geqslant \psi) \wedge(\varphi \geqslant \chi)) \rightarrow(\varphi \geqslant(\psi \vee \chi)) \quad \text { R } \quad \nabla \varphi \leftrightarrow \neg(\perp \geqslant \varphi)
\end{aligned}
$$

Figure 4 Logic WJR is $\mathbf{K}$ plus the above axiom schemas.

semantics, $\diamond \varphi$ is equivalent to $\neg(\perp \geqslant \varphi)$ (and hence $\varphi>\perp$, since $\varphi \geqslant \perp$ is valid). ${ }^{5}$

Unfortunately, as Yalcin $(2006,2009,2010)$ has demonstrated, this semantics based on Lewis's $l$-lifting validates some intuitively invalid patterns and fails to validate some intuitively valid patterns for 'at least as likely as'.

Fact 1 All of V1-V10, V12, and I1-I3, but neither V11 nor V13 (recall Figure 1), are valid over the class of world-ordering models with Lewis's $l$-lifting.

The fact that the $l$-lifting semantics validates I1 has become known as the "disjunction problem." Interestingly, I1 is the key axiom in a complete logic for this semantics.

Theorem 2 (Halpern 2003, Thm. 7.5.1a) The logic WJR in Figure 4 is sound and complete with respect to the class of world-ordering models with Lewis's $l$-lifting. 6,7

In light of the problems Yalcin raised with using Lewis's $l$-lifting for comparative likelihood, Kratzer (2012: 41) suggests a different method of lifting:

$$
A \succeq_{w}^{k} B \text { iff } \neg \exists b \in B_{w}-A_{w} \forall a \in A_{w}-B_{w}: b \succ_{w} a,
$$

where $b \succ_{w} a$ iff $b \succeq_{w} a$ and $a \succeq_{w} b$. However, as Lassiter (2013) observes, the $k$-lifting also leads to a disjunction problem: if the $\varphi$-worlds are disjoint from the $\psi \vee \chi$-worlds, the $\mathrm{J}$ axiom still holds, contrary to intuition in concrete examples.

As Kratzer (2012) notes, "which notions of comparative possibility provide the best match with natural language expressions relating to comparative modal notions related to probability... is still open" (42). We will return to this open question in $\$ 9$.

5 We can also define Kratzer's (1991) ' $\varphi$ is a good possibility' (which is true at a world $w$ in $\mathscr{M}$ iff $\left.\exists y \in R(w) \forall z \in R(w): z \succeq_{w} y \Rightarrow z \in \llbracket \varphi \rrbracket^{\mathscr{M}}\right)$ as $\neg(\neg \varphi \geqslant \top)$. However, we cannot define Kratzer's 'it must be that $\varphi^{\prime}\left(\forall x \in R(w) \exists y \in R(w): y \succeq_{w} x\right.$ and $\left.\forall z \in R(w): z \succeq_{w} y \Rightarrow z \in \llbracket \varphi \rrbracket^{\mathscr{M}}\right)$ in $\mathscr{L}$. It is noteworthy that if we substitute might $\varphi$ for $\nabla \varphi$ in Figures 4-5, then the axioms Mon and Ex are invalid according to Kratzer's (1991) semantics but valid according to Yalcin's (2010) semantics. As another example of the same phenomenon, the principle $(\varphi>\psi) \rightarrow$ might $\varphi$ is invalid according Kratzer's semantics but valid according to Yalcin's semantics. For related observations, see note 8. 6 Halpern's result is for the fragment of $\mathscr{L}$ without $\downarrow$, but it is a short step from there to Theorem 2. 7 The $\mathrm{D}$ axiom, $\square \varphi \rightarrow \diamond \varphi$, is derivable from Mon, BT, and $\mathbf{K}$. The D axiom is also derivable from all of the logics we consider later (see Figures 5 and 6), so we will not mention it again. 
Semantics for epistemic modals

$$
\begin{aligned}
\text { Ex } & \left(\square\left(\varphi \leftrightarrow \varphi^{\prime}\right) \wedge \square\left(\psi \leftrightarrow \psi^{\prime}\right)\right) \rightarrow\left((\varphi \geqslant \psi) \leftrightarrow\left(\varphi^{\prime} \geqslant \psi^{\prime}\right)\right) \\
\text { Bot } & \varphi \geqslant \perp \quad \text { BT } \neg(\perp \geqslant \top) \quad \text { Tot } \quad(\varphi \geqslant \psi) \vee(\psi \geqslant \varphi) \\
\operatorname{Scott}_{n} & \varphi_{1} \ldots \varphi_{n} \mathbb{E} \psi_{1} \ldots \psi_{n} \rightarrow\left(\left(\bigwedge_{i<n}\left(\varphi_{i} \geqslant \psi_{i}\right)\right) \rightarrow\left(\psi_{n} \geqslant \varphi_{n}\right)\right)
\end{aligned}
$$

Figure 5 Logic $\mathbf{F P}$ is $\mathbf{K}$ plus the axioms above; $\mathbf{F P}_{\infty}$ is given by the axioms of $\mathbf{F P}_{n}$ for all $n \in \mathbb{N}$; and $\mathbf{F P} \mathbf{P}_{\infty} \mathbf{R}$ is $\mathbf{F P}_{\infty}$ plus the axiom $\mathbf{R}$ in Figure 4.

\section{Finitely additive measure semantics}

Given the problems raised for Kratzer's semantics in §5, Yalcin (2010) and Lassiter Lassiter (2010) propose a measure semantics ${ }^{8}$ using models $\mathscr{M}=\langle W, R, V, \mathscr{F}, \mu\rangle$ as in $\S 4$ where for all $w \in W, \mu_{w}: \mathscr{F}_{w} \rightarrow[0,1]$ is finitely additive, i.e., for all $A, B \in \mathscr{F}_{w}$,

$$
\text { if } A \cap B=\emptyset \text {, then } \mu_{w}(A \cup B)=\mu_{w}(A)+\mu_{w}(B) \text {. }
$$

Finitely additive measure semantics avoids the problems with Kratzer's semantics.

Fact 2 All of V1-V13, and none of I1-I3, are valid over the class of finitely additive measure models.

The complete logic for finitely additive measure semantics was identified by Segerberg (1971) and Gärdenfors (1975), building on the work of Scott (1964).

Theorem 3 (Segerberg 1971; Gärdenfors 1975) The logic $\mathbf{F P}_{\infty}$ in Figure 5 is sound and complete with respect to the class of finitely additive measure models.

The notation $\varphi_{1} \ldots \varphi_{n} \mathbb{E} \psi_{1} \ldots \psi_{n}$ in $\operatorname{Scott}_{n}$ is an abbreviation for a long expression of $\mathscr{L}$, defined by Segerberg (1971: 342), which is true at a world $w$ iff for every

8 Although not at issue here, there is a difference between Yalcin and Lassiter's semantics for 'might' and 'possibly'. Yalcin $(2006,2007)$ considers a probabilistic semantics for 'might'/possibly' according to which: (i) might $\varphi /$ possibly $\varphi$ is true at $w$ iff $\mu_{w}\left(\llbracket \varphi \rrbracket^{\mathscr{M}}\right)>0$. However, his preferred semantics (Yalcin 2007, 2010) is equivalent to the semantics for $\diamond$ in $\S 2$, assuming the uniformity constraint on $R$ : (ii) might $\varphi /$ possibly $\varphi$ is true at $w$ iff $R(w) \cap \llbracket \varphi \rrbracket^{\mathscr{M}} \neq \emptyset$. Lassiter (2010: $\left.\S 4.4\right)$ adopts (i) as his semantics for possibly $\varphi$, and following Swanson (2006), Lassiter (2011: §3.8.2) adopts the following semantics for might $\varphi$ : (iii) might $\varphi$ is true at $w$ iff $\mu_{w}\left(\llbracket \varphi \rrbracket^{\mathscr{M}}\right)>\theta$ for a threshold $\theta$ (so must $\varphi$ is true at $w$ iff $\left.\mu_{w}\left(\llbracket \varphi \rrbracket^{\mathscr{M}}\right)>1-\theta\right)$. Note that the axioms Mon and Ex from Figures 4-5 and the principle $(\varphi>\psi) \rightarrow \diamond \varphi$ are valid according to (i) and (ii), but invalid according to (iii) (if $\theta>0$ ), in agreement with Kratzer might $\varphi$ (recall note 5). Also note that the principle $\diamond \varphi \rightarrow(\varphi>\perp)$ (cf. axiom R in Fig. 4) is valid according to (i) and (iii), but invalid according to (ii), and the right-to-left direction, $(\varphi>\perp) \rightarrow \diamond \varphi$, is valid according to (i) and (ii), but invalid according to (iii) (if $\theta>0$ ). 
world $v \in R(w)$, the same number of $\varphi_{i}$ 's are true at $v$ as $\psi_{i}$ 's, i.e.:

$$
\left|\left\{i \mid i \leq n, v \in \llbracket \varphi_{i} \rrbracket^{\mathscr{M}}\right\}\right|=\left|\left\{i \mid i \leq n, v \in \llbracket \psi_{i} \rrbracket^{\mathscr{M}}\right\}\right| .
$$

Using the definition of finite additivity, one can prove that all instances of the Scott schemata are valid over finitely additive measures. However, whether it is desirable for a semantics for 'at least as likely as' in ordinary language to validate these schemata is a different question, which we discuss in $\S 10$. As we show in $\S 7$, the Scott schemata are not necessary to derive the intuitively valid patterns V1-V13 in Figure 1. To appreciate the strength of the Scott schemata, it is noteworthy that they generate an infinite hierarchy of stronger and stronger logics, with $\mathbf{F P} \mathbf{P}_{\infty}$ at the top.

Theorem 4 (Scott \& Suppes 1958) For every $n \geq 1, \mathbf{F P}_{n+1}$ is a strictly stronger logic than $\mathbf{F P}$; in particular, not all instances of $\mathbf{S c o t t}_{n+1}$ are theorems of $\mathbf{F P} \mathbf{P}_{n}$.

Finally, for comparison with the semantics of $\S 9$, note that the axiom $\mathrm{R}$ in Figure 4 is valid over models in which for all $w \in W, \mu_{w}$ is regular in the sense that for all $A \in \mathscr{F}_{w}$, if $A \neq \emptyset$, then $\mu_{w}(A)>0$. The axiom $\mathrm{R}$ is equivalent to $\nabla \varphi \leftrightarrow(\varphi>\perp)$, the left-to-right direction of which is just the regularity condition. Indeed:

Theorem 5 The logic $\mathbf{F P} \mathbf{\infty}_{\infty} \mathbf{R}$ in Figure 5 is sound and complete with respect to the class of regular finitely additive measure models.

\section{Qualitatively additive measure semantics}

Let us consider a measure semantics with models $\mathscr{M}=\langle W, R, V, \mathscr{F}, \mu\rangle$, as in $\S 4$, where $\mu_{w}: \mathscr{F}_{w} \rightarrow[0,1]$ also satisfies what we will call qualitative additivity, the condition that for all $A, B \in \mathscr{F}_{w}$ :

$$
\mu_{w}(A) \geq \mu_{w}(B) \text { iff } \mu_{w}(A-B) \geq \mu_{w}(B-A),
$$

which corresponds to the axiom $(\varphi \geqslant \psi) \leftrightarrow((\varphi \wedge \neg \psi) \geqslant(\psi \wedge \neg \varphi))$.

Qualitative additivity is often stated in the equivalent form: for all $X, Y, Z \in \mathscr{F}_{w}$, if $(X \cup Y) \cap Z=\emptyset$, then $\mu_{w}(X) \geq \mu_{w}(Y) \Leftrightarrow \mu_{w}(X \cup Z) \geq \mu_{w}(Y \cup Z)$, which corresponds to the axiom $\neg \vee((\varphi \vee \psi) \wedge \chi) \rightarrow((\varphi \geqslant \psi) \leftrightarrow((\varphi \vee \chi) \geqslant(\psi \vee \chi)))$.

To see the motivation behind qualitative additivity, observe that the sentence (1) is intuitively equivalent to the simpler (2):

(1) It is at least as likely that [one of Brazil or Qatar will win the World Cup $]_{\varphi \vee \chi}$ as it is that [one of the U.S. or Qatar will win the World Cup] $]_{\psi \vee \chi}$.

(2) It is at least as likely that [Brazil will win the World Cup $]_{\varphi}$ as it is that [the U.S. will win the World Cup $]_{\psi}$. 
Semantics for epistemic modals

$$
\begin{aligned}
& \text { Ex }\left(\square\left(\varphi \leftrightarrow \varphi^{\prime}\right) \wedge \square\left(\psi \leftrightarrow \psi^{\prime}\right)\right) \rightarrow\left((\varphi \geqslant \psi) \leftrightarrow\left(\varphi^{\prime} \geqslant \psi^{\prime}\right)\right) \\
& \text { Bot } \quad \varphi \geqslant \perp \quad \text { BT } \neg(\perp \geqslant \top) \quad \text { Tot } \quad(\varphi \geqslant \psi) \vee(\psi \geqslant \varphi) \\
& \text { Tran } \quad(\varphi \geqslant \psi) \rightarrow((\psi \geqslant \chi) \rightarrow(\varphi \geqslant \chi)) \\
& \text { A } \quad(\varphi \geqslant \psi) \leftrightarrow((\varphi \wedge \neg \psi) \geqslant(\psi \wedge \neg \varphi))
\end{aligned}
$$

Figure 6 Logic FA is $\mathbf{K}$ plus the above axiom schemas.

Moreover, qualitatively additive measures pass all the checks from Figure 1.

Fact 3 All of V1-V13, and none of I1-I3, are valid over the class of qualitatively additive measure models.

As Theorem 6 shows, the class of qualitatively additive measures has a simple logic, the axioms of which appear intuitively justifiable. ${ }^{9}$ Indeed, the axioms of FA have formed the standard base system for "qualitative probability" since de Finetti 1949 (see, e.g., Fine 1973: §2; Fishburn 1986; Narens 2007: §4).

Theorem 6 The logic FA is sound and complete with respect to the class of qualitatively additive measure models (see Holliday \& Icard 2013b for a proof).

Interestingly, we can compare the logic FA of qualitatively additive measure models with the logic $\mathbf{F P}_{\infty}$ of finitely additive measure models as follows.

Fact 4 (van der Hoek 1996) The logic FA is equivalent to the logic $\mathbf{F P}_{3}$.

A question naturally arises from the foregoing results: what considerations about natural language could compel us beyond $\mathbf{F A} / \mathbf{F P} \mathbf{P}_{3}$ and qualitatively additive measures, all the way up to $\mathbf{F P}_{\infty}$ and finitely additive measures, for an adequate logic and semantics for 'at least as likely as' in English (as opposed to, say, for probability theory in science)? We will return to this methodological question in $§ 10$.

9 Tot may be an exception. Keynes 1921: $\$ 3$ is a classic source of arguments against totality and the numerical measurability of all propositions' probabilities. As Fine (1973: 18) notes, "the requirement that all events be comparable is not insignificant and has been denied by many careful students of probability including Keynes and Koopman.” Lassiter (2011: 81) suggests allowing incomparabilities in a semantics based on sets of measures, so $\varphi \geqslant \psi$ is true iff all measures in the set give as great a value to $\varphi$ as to $\psi$. For results on the sets-of-measures approach, see Holliday \& Icard 2013b. 
Holliday and Icard

\section{Event-ordering semantics}

We will not say much about the event-ordering semantics from $\S 4$ here, except for the obvious: one can obtain any logic for 'at least as likely as' that one likes by putting appropriate constraints on the orderings $\succsim_{w}$ of propositions. For example, the following are the semantic constraints corresponding to what we call the logic WA (Holliday \& Icard 2013b), which Gaifman (2009: 51) considers to be the "hard core for the logic of uncertain reasoning," obtained from FA by dropping Tot: ${ }^{10}$

$$
\begin{aligned}
& \text { (non-negativity) } A \succsim_{w} \emptyset \text {; (non-triviality) } \emptyset \succsim_{w} R(w) ; \\
& \text { (transitivity) if } A \succsim_{w} B \text { and } B \succsim_{w} C \text {, then } A \succsim_{w} C \text {; } \\
& \text { (qualitative additivity) } A \succsim_{w} B \text { iff } A-B \succsim_{w} B-A .
\end{aligned}
$$

We expect that some would object to this simple event-ordering semantics based on the view that a likelihood ordering on propositions should not be a primitive in the model, but rather should be derived from something else, such as a measure or a world ordering. ${ }^{11}$ But the question for the latter approach is: can an ordering on propositions with desirable properties be derived from an ordering on worlds?

\section{World-ordering semantics revisited}

Having seen in $\S 5$ that Lewis's (1973) method of lifting an ordering on worlds to an ordering on propositions (intended for comparative possibility) leads to undesirable results for comparative likelihood, let us consider Yalcin's (2010: 923) question: "Is there some better way of extending a preorder over worlds to a preorder over propositions, one which will get the inference patterns right?" Toward answering this question, observe that the $l$-lifting can be equivalently redefined as follows:

$$
A \succeq_{w}^{l} B \text { iff there is a function } f: B_{w} \rightarrow A_{w} \text { such that } \forall x \in B_{w}: f(x) \succeq_{w} x .
$$

Now consider the following modification of the $l$-lifting:

$A \succeq_{w}^{m} B$ iff there is an injection ${ }^{12} f: B_{w} \rightarrow A_{w}$ such that $\forall x \in B_{w}: f(x) \succeq_{w} x$.

According to the $l$-lifting, if there is one way that $A$ could happen that is ranked at least as highly as each of the ways that $B$ could happen, then $A$ is at least as

10 This shows the flexibility of event-ordering semantics: it is easy to drop totality from event-ordering semantics, whereas totality is built in to basic measure semantics. Recall note 9.

11 Why this should be so is an interesting issue, especially if one wants a purely qualitative semantics without numerical measures like Kratzer's.

12 Recall that a function $f: B \rightarrow A$ is an injection iff for all $x, y \in B, f(x)=f(y)$ implies $x=y$. 
Semantics for epistemic modals

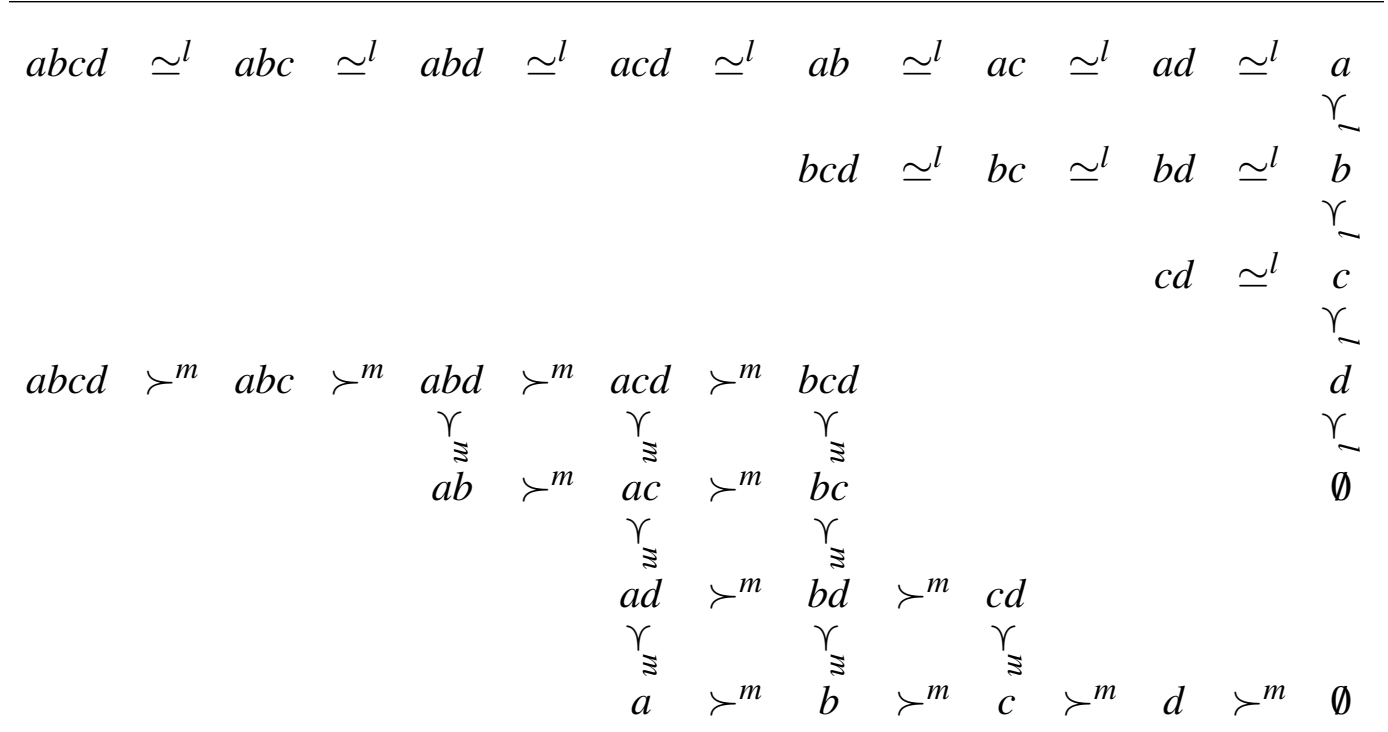

Figure 7 Comparison of Lewis's lifting $\succeq^{l}$ and the modified lifting $\succeq^{m}$ starting from $a \succ b \succ c \succ d$ (with relations implied by transitivity omitted).

likely as $B$-even if there are many more ways that B could happen, which is why the $l$-lifting gives the wrong results, e.g., the "disjunction problem." By contrast, according to the $m$-lifting, if every distinct way that $B$ could happen is matched by a distinct way that $A$ could happen that is at least as highly ranked, then $A$ is at least as likely as $B$; and this is the most information about the comparative likelihoods of propositions that we can extract from our information about the ranking of worlds.

While $\succeq_{w}^{l}$ is always a total relation if $\succeq_{w}$ is, this is not the case for $\succeq_{w}^{m}$, which may allow many incomparabilities. But this is how it should be, for there is simply not enough information in every total ordering on worlds to completely determine a total likelihood ordering on propositions. For example, if all we know is that $a \succ_{w} b \succ_{w} c \succ_{w} d$, then we know that $\{a, b\}$ is more likely than $\{b, c\}$, as both of the liftings imply; but we do not know whether $\{a\}$ is more likely than $\{b, c\}$. The $l$-lifting implies that $\{a\}$ is more likely than $\{b, c\}$, but jumping to this conclusion is a mistake; surely it is consistent with $a \succ_{w} b \succ_{w} c \succ_{w} d$ that $\{b, c\}$ is more likely than $\{a\}$. Fig. 7 shows the dramatically different results of applying the $l$-lifting and $m$-lifting to this world-ordering (with world-subscripts omitted).

While we leave open whether the $m$-lifting is a useful tool for semanticists in analyzing comparative epistemic modals, it is noteworthy that the predicted entailment patterns of this semantics are almost exactly the same as those of the 
(regular) finitely additive measure semantics from $\S 6,{ }^{13}$ except that the Tot axiom is not valid over world-ordering models with the $m$-lifting (recall note 9 ).

Fact 5 All of V1-V13, and none of I1-I3, are valid over the class of world-ordering models with the $m$-lifting.

Where $\mathbf{W P} \mathbf{P}_{\infty} \mathbf{R}$ is the logic obtained from $\mathbf{F P}_{\infty} \mathbf{R}$ by dropping Tot, we have:

Theorem 7 (Holliday \& Icard 2013a,b) The logic $\mathbf{W P} \mathbf{P}_{\infty} \mathbf{R}$ is sound-and $\mathbf{F P} \mathbf{P}_{\infty} \mathbf{R}$ is complete-with respect to the class of world-ordering models with the $m$-lifting.

Whether $\mathbf{W} \mathbf{P}_{\infty} \mathbf{R}$ is also complete we leave as an open technical question.

\section{Discussion}

Faced with a panoply of alternative semantic models, what might compel us toward adopting one over the others? Though we will not venture to answer this question here, we would like to discuss a number of issues that bear on the question.

\subsection{Semantic intuitions}

Recall the quotation from Chierchia \& McConnell-Ginet (2001) in §3. They state that the empirical base of semantics is our intuitive grasp of semantic properties and relations between expressions. As is well known from empirical work in psychology, if we apply this methodology too naïvely to expressions of comparative likelihood, we will end up with no logic to speak of and consequently no adequate modeltheoretic treatment. Tversky (1969) showed that in some cases subjects do not obey transitivity, and as Tversky \& Kahneman (1983) made famous, ordinary speakers do not even satisfy the basic monotonicity axiom (recall Figure 4), e.g., declaring $\varphi \wedge \psi$ to be strictly more likely than $\varphi$ alone. But transitivity and monotonicity are essential if we want any reasonable semantics based on measures, for example.

Suppose, however, that we can explain away these experimental results in some way or another. Indeed, suppose that on the basis of our intuitive grasp of entailments between sentences, we can convince ourselves that the logic of 'at least as likely as' is at least as strong as WA, or perhaps FA. ${ }^{14}$ The question we want to raise here is whether our basic semantic intuitions might be used to justify principles stronger than

13 It is also noteworthy that unlike the $l$-lifting, the $m$-lifting has the following property: if a worldordering $\succeq_{w}$ agrees with a measure $\mu_{w}$ in the sense that $v \succeq_{w} u \Leftrightarrow \mu_{w}(\{v\}) \geq \mu_{w}(\{u\})$, then the lifted ordering $\succeq_{w}^{m}$ almost agrees with $\mu_{w}$ in the sense that $A \succeq_{w}^{m} B \Rightarrow \mu_{w}(A) \geq \mu_{w}(B)$.

14 On a preliminary Mechanical Turk study, subjects unanimously agree with the equivalence between claims like (1) and (2) in $§ 7$, suggesting that principle A is intuitive at least in basic cases. 
A, the qualitative additivity principle. Recall (Fact 4) that FA is equivalent to $\mathbf{F P}_{3} .{ }^{15}$ What is the status of the "next strongest" system $\mathbf{F P}_{4}$ ? In particular, what would be an example of a prediction about natural language that would distinguish $\mathbf{F P}_{4}$ from FA? As it turns out, the answer to this question intersects with an interesting episode from the history of qualitative probability.

De Finetti, who famously held comparative probability judgments to be more fundamental than numerical probability judgments, once conjectured that the properties of orderings guaranteed by the logic FA would be sufficient to guarantee the existence of an agreeing finitely additive measure (de Finetti 1949), or in the terminology of the present work, that FA is complete with respect to finitely additive measure semantics. This was shown to be false by Kraft, Pratt \& Seidenberg (1959), who exhibited a particular ordering that is consistent with FA, but does not admit of an agreeing finitely additive measure and is therefore inconsistent with $\mathbf{F P}_{\infty}$. The example includes five worlds, $W=\{a, b, c, d, e\}$, and is given as follows: ${ }^{16}$

$$
\{a, e\} \succ\{c, d\} \quad\{b, c\} \succ\{a, d\} \quad\{d\} \succ\{a, c\} \quad\{a, c, d\} \succ\{b, e\} .
$$

Theorem 8 (Kraft et al. 1959) The ordering above, while consistent with FA, is inconsistent with $\mathbf{F P}_{\infty}$. Moreover, for every model with fewer than five worlds, FA and $\mathbf{F P} \mathbf{P}_{\infty}$ coincide, i.e., FA is sound on the model iff $\mathbf{F P}_{\infty}$ is sound on the model.

In fact, it is easy to see that this ordering is ruled out by Scott $_{4}$ and is thus not even consistent with $\mathbf{F P}_{4}$. Kraft et al. (1959) showed it is the simplest such ordering.

What does this mean from the perspective of natural language? It shows that the simplest examples distinguishing $\mathbf{F P}_{4}$ from $\mathbf{F A}$ will include at least five atomic propositions. Here is an example, following the theme of World Cup predictions:

(3) It is more likely that one of Argentina or England will win the World Cup than it is that one of China or Denmark will win.

(4) It is more likely that one of Brazil or China will win than it is that one of Argentina or Denmark will win.

(5) It is more likely that Denmark will win than it is that one of Argentina or China will win.

(6) It is more likely that one of Argentina, China, or Denmark will win than it is that one of Brazil or England will win.

To the extent that we find this combination of claims clearly inconsistent, that is evidence in favor of $\mathbf{F P}_{4}$, which rules it out as such. That is, if it is judged to be

15 Moreover, WA is equivalent to $\mathbf{W P}$. Most of what we say in $\S 10$ is neutral with regard to totality. 16 It is easy to extend this to a total ordering on $\wp(W)$. See, e.g., Fine 1973: 22. 
part of the meaning of 'at least as likely as' or 'more likely than' that (3)-(6) are inconsistent, then this would show that FA and its associated models are not strong enough to capture what these expressions mean. However, we find it doubtful that English speakers will have such strong intuitions about examples like this. Moreover, to repeat, this is the simplest kind of natural language example that could be used to motivate even the weakest of stronger systems. To motivate $\mathrm{Scott}_{5}, \mathrm{Scott}_{6}, \mathrm{Scott}_{7}$, and so on, up to $\mathbf{F P}_{\infty}$, would require even more complicated examples.

The challenge of motivating $\mathbf{F P} \mathbf{P}_{\infty}$ is different than the challenge of motivating, e.g., classical propositional logic. It is also true that there are complicated tautologies of classical logic that ordinary speakers would not recognize as intuitively valid. However, those complicated tautologies follow from simpler entailment principles that are (arguably) intuitively valid. By contrast, $\mathrm{Scott}_{4}, \mathrm{Scott}_{5}, \mathrm{Scott}_{6}$, and so on, do not seem to follow from simpler entailment principles (cf. Theorem 4). Other axioms of qualitative probability (most of which are not statable in $\mathscr{L}$ ) that are sufficient to guarantee compatibility with a finitely additive measure have been discussed (Krantz, Luce, Suppes \& Tversky 1971: §5), but we doubt that any of these conditions provide an easier route to justifying $\mathbf{F P}_{\infty}$ and finitely additive measure semantics based on intuitions about the ordinary meaning of 'at least as likely as'. ${ }^{17}$

It may be thought that combinations of statements like (3)-(6), while not obviously inconsistent or reducible to anything obviously inconsistent, are normatively objectionable upon closer scrutiny. ${ }^{18}$ After all, such statements are incompatible with any finitely additive measure, that is, incompatible with the Kolmogorov axioms for probability. One might attribute speakers' failure to reject (3)-(6) to processing limitations, while holding that (3)-(6) should be jointly inconsistent according to the semantics because their combination violates norms of reasoning.

Our first observation about this proposal is that it goes well beyond the methodology described by Chierchia \& McConnell-Ginet (2001), since it appeals to arguments about how one ought to reason, rather than more basic intuitions about, e.g., what follows from what. Second, and more importantly, it is by no means obvious that (3)-(6) are in any way objectionable, beyond merely not being compatible with finitely additive probability. We already mentioned Gaifman (2009), who considers principle A, but not Scott 4 or any stronger principle, to be part of the "hard core" for the logic of uncertain reasoning. Fine (1973: 23), in his seminal treatise on probability theory, argues that " $\mathrm{CP}$ [comparative probability] relations are not reasonably restricted to only those compatible with additive probability."

We certainly cannot hope to settle this issue here. Rather, we simply want to

17 This doubt also applies to the conditions listed in Theorem 3.5 of Krantz et al. 1971: 85 and Theorem 4.2 of Narens 2007: 33, which Lassiter (2011: 77) cites in an argument for finitely additive measure semantics (note especially Def. 3.4 of Krantz et al. 1971: 84 and Def. 4.5 of Narens 2007: 33).

18 On this question, see Icard 2013. 
Semantics for epistemic modals

call attention to the fact that these stronger logical principles, which correspond to the jump from qualitatively additive to finitely additive measures, seem to be far-removed from ordinary linguistic entailments and are even controversial from a mathematical, or normative, point of view. We tentatively conclude that if one wants to motivate finitely additive measure semantics over, say, qualitatively additive measure semantics, this cannot be done on the basis of intuitive entailments.

\subsection{Analogies: Heights and Times}

It may be instructive to consider what semanticists have said about analogous domains of discourse. Here we will briefly discuss discourse about time and height.

Just as in the case of epistemic modals, we can study the language of time by interpreting fragments in well-defined model classes. For talk about time this might include a basic "time scale," which is sometimes modeled with rational or real numbers. Already here, the structure of the basic time scale seems underdetermined by features of English or intuitions about what follows from what. For instance, the property of so-called Dedekind completeness, satisfied by the real numbers but not by the rational numbers, is captured by a formula that can be stated in a very simple language with only basic future ('it will be the case that', symbolized by $F$ ) and past ('it was the case that', symbolized by $P$ ) tense operators (van Benthem 1991): ${ }^{19}$

$$
(F H \varphi \wedge F \neg \varphi \wedge G(\neg \varphi \rightarrow G \neg \varphi)) \rightarrow F((\varphi \wedge G \neg \varphi) \vee(\neg \varphi \wedge H \varphi)) .
$$

While it is noteworthy that this property is expressible in such a simple languagejust as finite additivity is expressible in the language $\mathscr{L}$ using the Scott schematawe are not aware of any claim that this principle and its associated semantic property are properly part of the semantics of future and past tense.

Some authors have been reluctant to allow even more basic assumptions to figure into the semantics of temporal language. For instance, on the question of whether the time scale should be upper or lower bounded, Bach (1986: 579) rhetorically asks, "Are questions about the Big Bang and the Final Whimper linguistic questions?" Apparently our linguistic practices do not settle such matters. After all, these are questions of substantive scientific concern, and a number of linguists have explicitly denied the dependence of semantics on what science, or even tutored common sense, tells us about a given domain. For instance, Steedman (2010) writes:

As in any epistemological domain, neither the ontology nor the relations should be confused with the corresponding descriptors that we use to define the physics and mechanics of the real world. The

$19 G$ is an abbreviation for $\neg F \neg$, meaning 'it is always going to be the case that', and $H$ is an abbreviation for $\neg P \neg$, meaning 'it has always been the case that'. 
notion of time that is reflected in linguistic categories is only indirectly related to common-sense physics of clock-time and the related Newtonian representation of it as a dimension comprising an infinite number of instants corresponding to the real numbers, still less to the more abstruse representation of time in modern physics. (925)

This is in marked contrast to older views of semantics, according to which a semantic theory of some fragment just is a scientific theory of that fragment's subject matter. ${ }^{20}$

To relate this back to the semantics of epistemic modals, one could take the stance that the semantics of our talk about probability and likelihood should be determined by our best theory of probability and likelihood. Whether finitely additive probability is the best theory for comparative probability judgments is controversial, as noted. However, perhaps if one is convinced that it is, this may provide a route to justifying a strong logic like $\mathbf{F P}_{\infty}$ and finitely additive probability. One might think of this as a "regimentation" of our ordinary talk and reasoning. ${ }^{21}$ This would of course not be an empirical argument for such a strong logic, and in particular it would not be based on our ordinary semantic intuitions. This stance does seem coherent, but it is not the one generally taken in semantic theorizing about temporal language.

Some of the same themes come up in work on the semantics of gradable adjectives like 'tall'. At least since Lewis (1970), who attributes the idea to David Kaplan, a popular analysis of words like 'tall', and their comparative forms like 'taller than', is that they are interpreted in terms of scales. Analogous to the literature on qualitative probability, there is also a literature on qualitative axioms for extensive measurement (Krantz et al. 1971: §3). On one hand, we could explore a similar landscape of systems corresponding to stronger and stronger assumptions on scales for extensive quantities like height. On the other hand, theorists in this area have typically made as few assumptions as possible about properties of the underlying scale, thereby committing themselves to minimal logical validities. For instance, in an early paper on 'tall', Cresswell (1976: 266) writes, "It is tempting to think of $>$ as at least a partial ordering; whether it should be strict or not or total or not seems unimportant, and perhaps we should even be liberal enough not to insist on transitivity and antisymmetry." This is not to mention more substantive assumptions like additivity. Carrying this view over to comparative likelihood, we would be left with a logic much weaker than anything we have considered here. Such a view of semantics-as merely capturing the "logical forms" of sentences-is perhaps less

20 A classic statement of this view can be found in Bloomfield (1933: 139) chapter on semantics, where he says, "In order to give a scientifically accurate definition of meaning for every form of a language, we should have to have a scientifically accurate knowledge of everything in the speaker's world."

21 See, e.g., Eriksson \& Hájek (2007: 209), who argue that finitely additive probability "only adds structure and nuance to a concept. .. that we already had." 
popular than it once was. But it is one extreme worth mentioning. ${ }^{22}$

Even if one takes such an austere picture of what semantics ought to concern itself with, recent developments have motivated assuming at least some structure in scales for gradable adjectives. As Rotstein \& Winter (2004) and Kennedy \& McNally (2005) have convincingly argued, adjectives can be roughly classified on the basis of whether they can be combined with certain modifiers. These classes can be understood in terms of properties of scales. For instance, the scale for a given adjective is upper bounded just in case it is modifiable by such expressions as 'perfectly' or 'completely'. While 'perfectly flat' and 'completely flat' make sense, 'perfectly tall' and 'completely tall' do not. The scale for height is therefore assumed to be unbounded from above, as seems pretheoretically reasonable. ${ }^{23}$

There is a lively current debate about these issues for words such as 'likely' and 'probable' (Lassiter 2010; Klecha 2012). One question is whether they should be interpreted in terms of a closed scale. We do not wish to take a stand on this question. In fact, as far as the language $\mathscr{L}$ we have studied in this paper goes, the logic of 'at least as likely as' is neutral on this issue. ${ }^{24}$ However, it is an interesting question whether some analogous grammatical considerations could be used to argue for or against finite additivity. Compatibility with modifiers is not directly about entailment or validity, but it is nonetheless assumed to be relevant to the semantics of gradable adjectives. Could there be some such diagnostic relevant to finite additivity?

Summing up, in the domains of height and time, theoreticians have been reluctant to draw conclusions about semantics either from speakers' beliefs about the domain or from what the sciences tell us about the domain. It is an interesting open question whether there may be grammatical or other purely linguistic sources of evidence for or against finite additivity, for example, on analogy with scale boundedness. Absent such independent evidence, the insistence upon more restrictive classes of models and correspondingly stronger logical systems will have to be motivated by something other than first-order linguistic intuitions or behavior.

22 Wheeler (1972: 319) expresses an austere view: "Semantics, as we see it, is solely concerned with finding out what the forms of sentences in English are. When we have found where the predicates are, semantics is finished. It is certainly a worthwhile project, when semantics is done, to state some truths using the predicates the semantics has arrived at, but this is to do science, not semantics.... The tendency we oppose is the tendency to turn high-level truths into analytic truths."

23 Intriguingly, Kennedy (2007) suggests that our pretheoretic intuitions sometimes come apart from what is implicated in the semantics. One of his examples is '(in)expensive', which we might initially assume to imply a least degree of expense. However, because 'barely expensive' and 'completely inexpensive' seem infelicitous, he argues that the scale ought to be open. Thus, grammatical considerations are claimed to eclipse pretheoretic intuitions about the domain in question.

24 We assumed a closed scale from 0 to 1 for reasons of convenience and custom. 
Holliday and Icard

\section{Conclusion}

In this paper, we have considered a number of semantic accounts and associated logics for the comparative epistemic modal 'at least as likely as'. We have focused on semantics that give rise to the logics FA or WA, with models based on qualitatively additive measures or qualitative event-orderings, and the logics $\mathbf{F P} \mathbf{P}_{\infty}$ or $\mathbf{W} \mathbf{P}_{\infty} \mathbf{R}$, with models based on finitely additive measures or qualitative world-orderings. As we have seen, there is a large gap to bridge between $\mathbf{F A} / \mathbf{W A}$ and $\mathbf{F P} \mathbf{P}_{\infty} / \mathbf{W P} \mathbf{P}_{\infty} \mathbf{R}$. Indeed, the question of how to motivate going beyond de Finetti's logic FA to the logic $\mathbf{F P}_{\infty}$ of finitely additive probability has been difficult to answer even for probability theorists and philosophers of probability. It would be remarkable if the gap between FA and $\mathbf{F P}_{\infty}$ could be bridged by considerations from natural language.

\section{References}

Bach, Emmon. 1986. Natural language metaphysics. In R. Barcan Marcus, G.J.W. Dorn \& P. Weingartner (eds.), Logic, Methodology and Philosophy of Science VII, 573-595. Amsterdam: North-Holland.

van Benthem, Johan. 1991. The Logic of Time: A Model-Theoretic Investigation into the Varieties of Temporal Ontology and Temporal Discourse. Dordrecht: Kluwer Academic Publishers 2nd edn.

Bloomfield, Leonard. 1933. Language. New York: Henry Holt.

Chellas, Brian F. 1980. Modal Logic: An Introduction. New York: Cambridge University Press.

Chierchia, Gennaro \& Sally McConnell-Ginet. 2001. Meaning and Grammar. Cambridge, Mass.: MIT Press 2nd edn.

Cresswell, Max. 1976. The semantics of degree. In B.H. Partee (ed.), Montague Grammar, 261-292. New York: Academic Press.

Eriksson, Lina \& Alan Hájek. 2007. What are degrees of belief? Studia Logica 86(2). 183-213.

Fine, Terrence L. 1973. Theories of Probability. New York: Academic Press.

de Finetti, Bruno. 1949. La 'logica del plausible' secondo la concezione di Polya. Atti della XLII Riunione, Societa Italiana per il Progresso delle Scienze 227-236.

Fishburn, Peter C. 1986. The axioms of subjective probability. Statistical Science 1(3). 335-358.

Gaifman, Haim. 2009. Interview. In A. Hajék \& V. F. Hendricks (eds.), Probability and Statistics: 5 Questions, Copenhagen: Automatic Press / VIP.

Gärdenfors, Peter. 1975. Qualitative probability as an intensional logic. Journal of Philosophical Logic 4(2). 171-185. 
Semantics for epistemic modals

Halpern, Joseph Y. 2003. Reasoning about Uncertainty. Cambridge, Mass.: MIT Press.

Hamblin, Charles L. 1959. The modal "probably". Mind 68(270). 234-240.

van der Hoek, Wiebe. 1996. Qualitative modalities. International Journal of Uncertainty, Fuzziness, and Knowledge-Based Systems 4(1). 45-59.

Holliday, Wesley H. \& Thomas F. Icard. 2013a. Extending a comparative likelihood relation on a set to the powerset. Manuscript.

Holliday, Wesley H. \& Thomas F. Icard. 2013b. On the logic of comparative likelihood. Manuscript.

Icard, Thomas F. 2013. Pragmatic considerations on comparative probability. Manuscript.

Kennedy, Chris. 2007. Vagueness and grammar: The semantics of relative and absolute gradable adjectives. Linguistics and Philosophy 30(1). 1-45.

Kennedy, Chris \& Louise McNally. 2005. Scale structure and the semantic typology of gradable predicates. Language 81(2). 345-381.

Keynes, John Maynard. 1921. A Treatise on Probability. London: The Macmillan Company.

Klecha, Peter. 2012. Positive and conditional semantics for gradable modals. In I. Reich, E. Horch \& D. Pauly (eds.), Sinn \& Bedeutung 16, 363-376.

Koopman, B.O. 1940. The Axioms and Algebra of Intuitive Probability. Annals of Mathematics 41(2). 269-292.

Kraft, Charles H., John W. Pratt \& A. Seidenberg. 1959. Intuitive probability on finite sets. The Annals of Mathematical Statistics 30(2). 408-419.

Krantz, David H., R. Duncan Luce, Patrick Suppes \& Amos Tversky. 1971. Foundations of Measurement, vol. 1. New York: Academic Press.

Kratzer, Angelika. 1991. Modality. In A. von Stechow \& D. Wunderlich (eds.), Semantics: An International Handbook of Contemporary Research, 639-650. Berlin: de Gruyter.

Kratzer, Angelika. 2012. Modals and Conditionals. Oxford University Press.

Lassiter, Daniel. 2010. Gradable epistemic modals, probability, and scale structure. In N. Li \& D. Lutz (eds.), Semantics and Linguistic Theory (SALT) 20, 1-18. CLC (Cornell Linguistics Circle).

Lassiter, Daniel. 2011. Measurement and modality: The scalar basis of modal semantics. New York University PhD dissertation.

Lassiter, Daniel. 2013. Epistemic comparison, models of uncertainty, and the disjunction puzzle. Manuscript.

Lewis, David. 1970. General semantics. Synthese 22. 18-67.

Lewis, David. 1973. Counterfactuals. Oxford: Basil Blackwell.

Lewis, David. 1981. Ordering semantics and premise semantics for counterfactuals. Journal of Philosophical Logic 10(2). 217-234. 
Narens, Louis. 2007. Theories of Probability: An Examination of Logical and Qualitative Foundations. London: World Scientific Publishing.

Rotstein, Carmen \& Yoad Winter. 2004. Total adjectives vs. partial adjectives: scale structure and higher-order modification. Natural Language Semantics 12. 259-288.

Scott, Dana. 1964. Measurement structures and linear inequalities. Journal of Mathematical Psychology 1. 233-247.

Scott, Dana \& Patrick Suppes. 1958. Foundational aspects of theories of measurement. Journal of Symbolic Logic 23. 113-128.

Segerberg, Krister. 1971. Qualitative probability in a modal setting. In E. Fenstad (ed.), Second Scandinavian Logic Symposium, 341-352. Amsterdam: NorthHolland.

Steedman, Mark. 2010. Temporality. In J. van Benthem \& A. ter Meulen (eds.), Handbook of Logic and Language, 925-970. Amsterdam: Elsevier 2nd edn.

Swanson, Eric. 2006. Interactions with context. Massachusetts Institute of Technology $\mathrm{PhD}$ dissertation.

Tversky, Amos. 1969. Intransitivity of preferences. Psychological Review 76(1). 31-48.

Tversky, Amos \& Daniel Kahneman. 1983. Extensional versus intuitive reasoning: The conjunction fallacy in probability judgment. Psychological Review 90(4). 293-315.

Wheeler, Samuel C. 1972. Attributes and their modifiers. Noûs 6(4). 310-334.

Yalcin, Seth. 2006. Notes on epistemic modals. Talk at the University of Massachusetts, Amherst, Department of Linguistics, 10/12/2006.

Yalcin, Seth. 2007. Epistemic modals. Mind 116(464). 983-1026.

Yalcin, Seth. 2009. The language of probability. Talk at the UC Berkeley Linguistics Colloquium, 11/30/2009.

Yalcin, Seth. 2010. Probability operators. Philosophy Compass 5(11). 916-937.

Wesley H. Holliday

Department of Philosophy

314 Moses Hall \#2390

University of California

Berkeley, CA 94720-2390

wesholliday@berkeley.edu
Thomas F. Icard, III

Department of Philosophy

Building 90

Stanford University

Stanford, CA 94305-2155

icard@stanford.edu 